\title{
Association Between Risk Factors and Intensive Nutritional Intervention Outcomes in Elderly Individuals
}

\author{
Masakazu Hiramatsu ${ }^{\mathrm{a}, \mathrm{f}}$, Chika Momoki ${ }^{\mathrm{b}}$, Yumi Oide ${ }^{\mathrm{c}}$, Chiduko Kaneishic, \\ Yoko Yasui ${ }^{\mathrm{d}}$, Kumiko Shojia ${ }^{\mathrm{a}}$, Takashi Fukuda ${ }^{\mathrm{e}}$, Daiki Habu ${ }^{\mathrm{a}}$
}

\begin{abstract}
Background: The purpose of this study was to identify risk factors for intensive nutritional intervention outcomes in elderly undernourished patients to help reduce the number of patients with prolonged hospital stay or without recuperation of previous activities of daily living and quality of life.
\end{abstract}

Methods: In total, 230 patients who received interventions from a nutrition support team (NST) between January 2016 and July 2018 were included. Patients were classified into two groups based on NST intervention outcomes: patients with improved nutritional status were included in the successful group, whereas those whose nutritional status did not improve, as defined by progressive illness or death, were classified into the non-successful group. We assessed patient characteristics, laboratory data, and nutrition support methods.

Results: Our multivariate Cox proportional hazard analysis showed that: 1) The presence of peripheral parenteral nutrition (hazard ratio (HR): 1.80; 95\% confidence interval (CI): 1.13 - 2.88) was identified as an independent risk factor for NST intervention outcomes; 2) The energy fill rate to total energy expenditure was $<66.0 \%$ (HR: 1.61 ; 95\% CI: 0.98 - 2.66); and 3) A geriatric nutritional risk index score $<$ 70.0 (HR: 1.54 ; $95 \%$ CI: $0.92-2.56$ ) tended to be negatively associ-

Manuscript submitted January 3, 2019, accepted April 29, 2019

a Department of Medical Nutrition, Graduate School of Human Life Science, Osaka City University, 3-3-138 Sugimoto, Sumiyoshi-ku, Osaka-shi, Osaka 558-8585, Japan

${ }^{b}$ Department of Food and Nutrition, Faculty of Contemporary Human Life Science, Tezukayama University, 3-1-3 Gakuennminami, Nara-shi, Nara 6318585, Japan

'Department of Nutrition, Minami Osaka Hospital, 1-18-18 Higashikagaya, Suminoe-ku, Osaka-shi, Osaka 559-0012, Japan

dDepartment of Clinical Nutrition, Graduate School of Human Life Science, Osaka City University, 3-3-138 Sugimoto, Sumiyoshi-ku, Osaka-shi, Osaka 558-8585, Japan

eDepartment of Gastroenterological Medicine, Minami Osaka Hospital, 1-1818 Higashikagaya, Suminoe-ku, Osaka-shi, Osaka 559-0012, Japan

${ }^{f}$ Corresponding Author: Masakazu Hiramatsu, Department of Medical Nutrition, Graduate School of Human Life Science, Osaka City University, 3-3-138 Sugimoto, Sumiyoshi-ku, Osaka-shi, Osaka 558-8585, Japan.

Email: m_t_k_n_m_s_57@yahoo.co.jp

doi: https://doi.org/10.14740/jocmr3738 ated with NST intervention outcomes.

Conclusions: In addition to the nutrition therapy provided by a traditional NST, patients with the risk factors require nutritional intervention. Elderly individuals should also receive nutrition care because they have been recuperating at their home or in long-term care facilities, to prevent experiencing adverse conditions.

Keywords: Elderly; Energy fill rate; Geriatric nutritional risk index; Malnutrition; Nutrition support team; Nutritional assessment; Peripheral parenteral nutrition; Undernourished

\section{Introduction}

Elderly individuals who are recuperating at their homes, nursing homes, or in long-term care facilities are often admitted to acute-care hospitals because of bone fractures, pneumonia, anorexia, and relapse of previous illnesses. Seriously ill patients, who require hospitalization or surgery, are admitted in acutecare hospitals, and the length of stay in this type of hospital is limited to approximately 2 weeks. Nutrition intervention in parallel with medical treatment is, thus, very important for successful acute medical care for elderly patients and their early discharge from the hospital after remission. Because undernutrition in elderly individuals is associated with a reduction in activities of daily living (ADLs) or quality of life (QOL), the incidence of complications, mortality rate [1-3], and undernutrition in elderly patients is high $(30-60 \%)$ in acute care wards [4]. In Japan, nutrition support teams (NSTs) that consist of professionals including a doctor and a registered dietitian, who provide intensive nutrition therapy for undernourished patients, are widespread. NSTs have been initiated with the development of total parenteral nutrition (TPN) by Dudrick et al in 1968 [5]. The effects of NST interventions have been reported [6-10]. For example, the interventions helped to improve nutrition assessment, therapy, and patients' nutrition status, decreased the number of complications caused by enteral or parenteral nutrition, shortened hospitalization, and reduced medical expenses.

We noted that NST contributes to medical therapy in acute-care hospitals as part of the comprehensive care provided for elderly individuals. We analyzed the characteristics of patients whose nutrition status did not improve following 


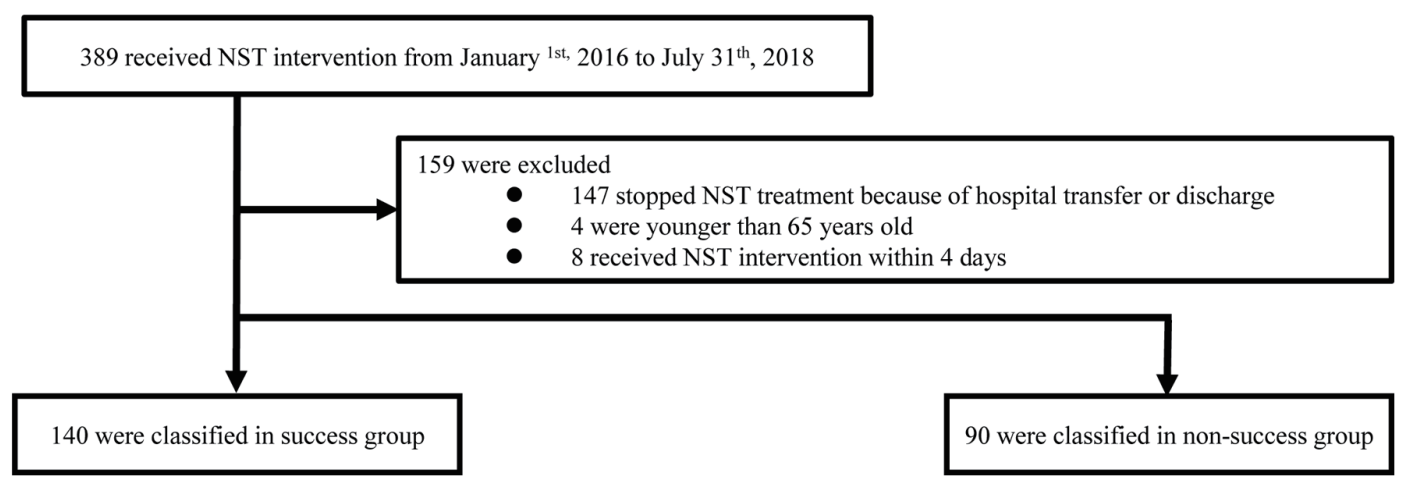

Figure 1. Flow chart of participant selection in this study.

intensive nutrition therapy from an NST with the aim to help reduce the number of patients with prolonged hospital stay or without recuperation of previous ADLs and QOL because of undernutrition.

Therefore, the purpose of this study was to prospectively identify non-successful factors (risk factors) associated with intensive nutritional intervention outcomes of an NST in elderly undernourished patients.

\section{Materials and Methods}

\section{Participants}

Registered dietitians in the hospital identified patients who met the following criteria (two or more of the factors): 1) Dietary intake $\leq 50.0 \%$; 2) Serum albumin (Alb) $\leq 2.7 \mathrm{~g} / \mathrm{dL}$; and (3) Body mass index $(\mathrm{BMI}) \leq 18.5 \mathrm{~kg} / \mathrm{m}^{2}$. Terminally ill patients who were not clinically indicated for NST intervention, as determined by a primary care provider, were excluded. Patients clinically indicated for NST intervention, as determined by a primary care provider, were included. These groups comprised the total cohort of potential study participants.

In total, 389 patients who received NST intervention in Minami Osaka Hospital in Osaka City, Japan, from January 1, 2016 to July 31, 2018, were enrolled in the study. Patients who discontinued NST treatment because of hospital transfer or discharge owing to financial reasons before receiving a final NST evaluation (147 patients), those younger than 65 years (four patients), and those who received care from the NST for less than 4 days (eight patients) were excluded from the study. In total, 230 patients participated in the study. Subsequently, patients were classified into two groups based on NST intervention outcomes (the successful group and non-successful group) (Fig. 1).

This study was conducted in accordance with the Declaration of Helsinki and was approved by the ethics committee of Osaka City University (no. 16-35).

\section{NST intervention outcomes}

The NST chairman (doctor) finally determined the NST inter- vention outcomes, after meeting with the NST staff, by reference to objective parameters, including the energy fill rate to total energy expenditure (TEE) $>70.0 \%$ or serum Alb $>2.6 \mathrm{~g} /$ dL. Severely undernourished patients whose nutrition status improved and those who did not require intensive nutritional care by the NST were classified under the successful group, while those whose nutrition status did not improve because of progressive disease or death were included in the non-successful group.

\section{Characteristics of the participants}

The clinical data, including sex, age, BMI, Charlson Comorbidity Index (CCI) score [11], subjective global assessment (SGA) score, the presence/absence of dysphagia and bedsore, the duration of NST intervention, and the energy fill rate to TEE (\%TEE), were analyzed.

BMI was calculated based on the patient's height and weight. The type and level of severity of comorbidities in the participants were evaluated using the CCI. We classified the participants into four groups based on the CCI score: low (0 point), medium ( 1 - 2 points), high ( 3 - 4 points), and very high ( $\geq 5$ points). The SGA scores were comprehensively scored such as physical items, food intake, and laboratory data. We classified the participants with an SGA score of 0 as having normal nutritional condition; 1 - 2 points, light malnutrition; 3 - 4 points, moderate malnutrition; and 5 - 10 points, severe malnutrition. An experienced speech therapist assessed the presence/absence of dysphagia. \% TEE was calculated using the following formula: \% TEE = (total energy intakes $(\mathrm{kcal}) /($ basal metabolic rate $(\mathrm{kcal}) \times$ activity factor $\times$ stress factor $) \times 100$ [12]. Basal metabolic rate was calculated using a simple Japanese formula [13]: men: 14.1 $\times$ body weight $(\mathrm{kg})+620$; women: $10.8 \times$ body weight $(\mathrm{kg})$ +620 .

\section{Laboratory examinations}

At the time of NST enrollment, we measured the level of Alb, total lymphocyte count (TLC), total cholesterol (T-Cho), Creactive protein (CRP), transthyretin (TTR), hemoglobin $(\mathrm{Hb})$, 
and triglyceride (TG).

We calculated the controlling nutritional status (CONUT) $[14,15]$, Onodera's prognostic nutritional index (PNI) [16], and geriatric nutritional risk index (GNRI) scores [17]. The CONUT score was calculated using the Alb, TLC, and T-Cho levels. The PNI and GNRI scores were calculated using the following formulas: $\mathrm{PNI}=10 \times \mathrm{Alb}(\mathrm{g} / \mathrm{dL})+0.005 \times \mathrm{TLC}$ $(/ \mu \mathrm{L})[16] ; \mathrm{GNRI}=1.489 \times \mathrm{Alb}(\mathrm{g} / \mathrm{dL}) \times 10+41.7 \times($ body weight $(\mathrm{kg}) /$ ideal body weight $(\mathrm{kg}))$ [17].

\section{Methods used to supply nutrients}

The methods for supplying nutrients were classified into seven groups: TPN, peripheral parenteral nutrition (PPN), nasal feeding, gastric fistula (percutaneous endoscopic gastrostomy), regular texture diet (including therapeutic diet), oral supplementation, and dysphagia diet. Oral supplementation included nutrient-rich (carbohydrates, proteins, or trace elements) food, such as juice, ice cream, and jellies; protein-modified rice cracker or cookie; yogurt; or enteral nutrients. We documented the methods used for supplying nutrients to each patient at the time of NST enrollment.

\section{Assessment of potential risk factors and determination of cut-off values}

Prior to this study, we retrospectively collected data from patients treated in 2014 to predict NST intervention outcomes and identify the cut-off values of the extracted quantitative variable via an analysis of the receiver operating characteristic (ROC) curves. Factors, such as \%TEE; Alb, TLC, CRP, and TTR levels; CONUT, PNI, and GNRI score; and the presence/absence of PPN, were significantly different between the successful and non-successful groups. An analysis of the ROC curves was performed to obtain the cut-off values of the quantitative variables other than the presence/absence of PPN, area under the curve (AUC), and 95\% confidence interval (CI). The cut-off values for each factor were as follows: \%TEE, 66.0\% (AUC: 0.63; 95\% CI: 0.51 - 0.74); Alb level, $2.4 \mathrm{~g} / \mathrm{dL}$ (AUC: $0.72 ; 95 \%$ CI: $0.61-0.82$ ); TLC, $1,195 / \mu \mathrm{L}$ (AUC: $0.68 ; 95 \%$ CI: $0.57-0.80$ ); CRP level, $2.00 \mathrm{mg} / \mathrm{dL}$ (AUC: $0.68 ; 95 \%$ CI: $0.57-0.79$ ); TTR level, $8.5 \mathrm{mg} / \mathrm{dL}$ (AUC: $0.73 ; 95 \%$ CI: 0.60 - 0.87); CONUT score, 8 (AUC: 0.67 ; $95 \%$ CI: 0.55 - 0.79); PNI score, 33.0 (AUC: $0.75 ; 95 \%$ CI: $0.65-0.85$ ); and GNRI score, 70.0 (AUC: $0.75 ; 95 \%$ CI: $0.64-0.87)$.

\section{Statistical analysis}

Data were expressed as median (25th - 75th percentile) or percentage (numbers). In the successful and non-successful groups, the quantitative variables were compared using the Mann-Whitney U test, and the categorical variables were compared using Fisher's exact test or Chi-square test.

Univariate and multivariate Cox proportional hazard anal- yses were performed to identify the risk factors associated with NST intervention outcomes and to obtain the crude and adjusted hazard ratio (HR) and 95\% CI. Age; sex; BMI; CCI, SGA, CONUT, PNI, and GNRI scores; \%TEE; Alb, TLC, CRP, and TTR levels; and the presence/absence of PPN were considered as potential risk factors. The quantitative variables were classified into two groups. Age, BMI, CCI, and SGA score were divided as follows: age $\geq 85$ or $<85$ years, $\mathrm{BMI} \geq 18.5$ or $<$ $18.5 \mathrm{~kg} / \mathrm{m}^{2}, \mathrm{CCI} \geq 2$ or $<2$ points, and SGA score $\geq 3$ or $<3$ points. The cut-off values of CCI and SGA are shown as median. \%TEE, Alb, TLC, CRP, and TTR levels and CONUT, PNI, and GNRI scores were classified based on the cut-off values. In the multivariate Cox proportional hazard analysis, factors, such as age; sex; CCI, SGA, and GNRI scores; TLC and CRP levels; the presence/absence of PPN; and \%TEE, were selected based on the results of the univariate Cox proportional hazard analysis.

IBM $^{\circledR}$ SPSS ${ }^{\circledR}$ Statistics 23 software (IBM Japan, Tokyo, Japan) was used for the analysis, except for the univariate and multivariate Cox proportional hazard analysis, which were performed with SAS 9.4 (SAS Institute Inc., Cary, NC, USA). A P value less than 0.05 was considered statistically significant.

\section{Results}

\section{Characteristics of the participants}

The characteristics of the participants at the time of NST enrollment are summarized in Table 1. The percentage of men was significantly higher in the non-successful group than in the successful group. \%TEE was significantly lower in the nonsuccessful group than in the successful group. The duration of NST intervention was significantly shorter in the non-successful group than in the successful group. Participants in both groups were extremely old. The median age of the successful group was 85.0 years, and that of the non-successful group was 84.0 years. Age, BMI, CCI and SGA scores, and the presence/ absence of dysphagia and bedsore were not significantly different between the two groups.

\section{Laboratory examination results}

The results of the laboratory examinations at the time of NST enrollment are shown in Table 2. The Alb and TTR levels and PNI and GNRI scores were significantly lower in the nonsuccessful group than in the successful group. The CRP level was significantly higher in the non-successful group than in the successful group. The TLC, T-Cho, Hb, and TG levels and CONUT score were not significantly different between the two groups.

\section{Methods used to supply nutrients}

Table 3 shows the methodology used to supply nutrients 
Table 1. Characteristics of the Participants

\begin{tabular}{|c|c|c|c|c|c|}
\hline \multirow[b]{2}{*}{ Age (years) } & \multicolumn{2}{|c|}{ Successful group $(n=140)$} & \multicolumn{2}{|c|}{ Non-successful group $(n=90)$} & \multirow{2}{*}{$\begin{array}{l}\text { P value } \\
0.426\end{array}$} \\
\hline & 85.0 & $(79.0-89.0)$ & 84.0 & $(78.8-89.0)$ & \\
\hline Sex (men) & $35.0 \%$ & (49) & $51.1 \%$ & $(46)$ & 0.020 \\
\hline Lack of data & & (3) & & (5) & \\
\hline \multicolumn{6}{|l|}{ CCI (points) } \\
\hline High, 3 - 4 & $20.7 \%$ & (29) & $18.9 \%$ & $(17)$ & \\
\hline Very high, $\geq 5$ & $15.0 \%$ & $(21)$ & $23.3 \%$ & $(21)$ & \\
\hline \multicolumn{6}{|l|}{ SGA score (points) } \\
\hline Normal, 0 & $11.4 \%$ & $(15)$ & $5.8 \%$ & $(5)$ & 0.102 \\
\hline Lack of data & & $(8)$ & & (4) & \\
\hline Presence of dysphagia & $48.9 \%$ & $(68)$ & $58.9 \%$ & $(53)$ & 0.175 \\
\hline Lack of data & & $(1)$ & & $(0)$ & \\
\hline Presence of bedsore & $11.4 \%$ & $(15)$ & $17.4 \%$ & $(15)$ & 0.230 \\
\hline Lack of data & & $(8)$ & & (4) & \\
\hline$\%$ TEE $(\%)$ & 66.0 & $(44.8-82.1)$ & 56.3 & $(36.2-73.2)$ & 0.004 \\
\hline Duration of NST intervention (days) & 36.0 & $(22.0-50.0)$ & 22.0 & $(14.0-43.0)$ & $<0.001$ \\
\hline
\end{tabular}

Data are expressed as median (25th - 75th percentile) or percentage $(n)$. The differences between the two groups were analyzed using the Fisher's exact test, Chi-square test, and Mann-Whitney U test. BMI: body mass index; CCl: Charlson Comorbidity Index; SGA: subjective global assessment; \%TEE: the energy fill rate to total energy expenditure; NST: nutrition support team.

at the time of NST enrollment. The rate of PPN was significantly higher in the non-successful group than in the successful group. The rates of TPN, nasal feeding, gastric fistula, regular texture diet, oral supplementation, and dysphagia diet were not significantly different between the two groups.

Table 2. Laboratory Examination Results

\begin{tabular}{|c|c|c|c|c|c|c|c|}
\hline & \multicolumn{3}{|c|}{ Successful group $(n=140)$} & \multicolumn{3}{|c|}{ Non-successful group $(n=90)$} & \multirow{2}{*}{ P value } \\
\hline & $\mathrm{n}$ & & & $\mathrm{n}$ & & & \\
\hline Alb level (g/dL) & 140 & 2.4 & $(2.0-2.7)$ & 90 & 2.2 & $(1.8-2.6)$ & 0.004 \\
\hline T-Cho level (mg/dL) & 122 & 137 & $(106-160)$ & 76 & 133 & $(102-161)$ & 0.771 \\
\hline CRP level (mg/dL) & 139 & 2.16 & $(0.83-4.80)$ & 90 & 4.25 & $(1.39-9.08)$ & 0.001 \\
\hline Hb level $(g / d L)$ & 139 & 9.2 & $(8.4-10.5)$ & 90 & 9.2 & $(8.3-10.6)$ & 0.930 \\
\hline TG level (mg/dL) & 122 & 90 & $(63-111)$ & 69 & 89 & $(66-116)$ & 0.607 \\
\hline CONUT score & 122 & 8.0 & $(6.0-10.0)$ & 76 & 8.5 & $(7.0-10.0)$ & 0.304 \\
\hline PNI score & 136 & 29.6 & $(25.8-34.5)$ & 86 & 27.2 & $(23.3-32.5)$ & 0.017 \\
\hline GNRI score & 137 & 72.0 & $(64.3-78.2)$ & 85 & 67.8 & $(61.0-73.4)$ & 0.003 \\
\hline
\end{tabular}

Data are expressed as median (25th - 75th percentile). The differences between the two groups were analyzed using the Mann-Whitney $U$ test. Alb: albumin; TLC: total lymphocyte count; T-Cho: total cholesterol; CRP: C-reactive protein; TTR: transthyretin; Hb: hemoglobin; TG: triglyceride; CONUT: controlling nutritional status; PNI: Onodera's prognostic nutritional index; GNRI: geriatric nutritional risk index. 
Table 3. Methods Used to Supply Nutrients at the Time of NST Enrollment

\begin{tabular}{|c|c|c|c|c|c|}
\hline & \multicolumn{2}{|c|}{ Successful group $(n=140)$} & \multicolumn{2}{|c|}{ Non-successful group $(n=90)$} & \multirow{2}{*}{$\begin{array}{l}\text { P value } \\
0.173\end{array}$} \\
\hline Total parenteral nutrition & $16.4 \%$ & $(23)$ & $24.4 \%$ & $(22)$ & \\
\hline Peripheral parenteral nutrition & $32.1 \%$ & $(45)$ & $47.8 \%$ & (43) & 0.019 \\
\hline Gastric fistula (PEG) & $6.4 \%$ & (9) & $2.2 \%$ & (2) & 0.209 \\
\hline Regular texture diet (including therapeutic diet) & $50.7 \%$ & $(71)$ & $46.7 \%$ & $(42)$ & 0.590 \\
\hline Dysphagia diet & $35.7 \%$ & $(50)$ & $41.1 \%$ & (37) & 0.486 \\
\hline
\end{tabular}

Data are expressed as percentage (n). The differences between the two groups were analyzed using the Fisher's exact test. PEG: percutaneous endoscopic gastrostomy.

\section{Results of the Cox proportional hazard analysis}

Table 4 shows the results of the univariate and multivariate Cox proportional hazard analyses, which were performed to determine the risk factors associated with NST intervention outcomes. The unadjusted univariate Cox proportional hazard analysis suggested that \%TEE; Alb, CRP, and TTR levels; GNRI score; and PPN were significantly associated with NST intervention outcomes, while SGA and PNI scores showed just a tendency for association. In the multivariate Cox proportional hazard analysis, which was adjusted for age; sex; CCI, SGA, and GNRI scores; \%TEE, TLC and CRP levels; and PPN, the presence of PPN (HR: 1.80; 95\% CI: 1.13 - 2.88) was identified as an independent risk factor associated with NST intervention outcomes; moreover, \% TEE < 66.0\% (HR: 1.61; 95\% CI: 0.98 - 2.66) and GNRI score < 70.0 (HR: 1.54; 95\% CI: 0.92 - 2.56) tended to be negatively associated with NST intervention outcomes. Alb and TTR levels and PNI score were associated with NST intervention outcomes based on the univariate analysis. However, these factors had a significant correlation with GNRI score. Therefore, these factors were excluded from the multivariate Cox proportional hazard analysis to avoid collinearity. Age, sex, BMI, TLC level, and CCI, SGA and CONUT scores were not associated with NST intervention outcomes.

\section{Discussion}

In our previous study, which included 101 elderly patients who received NST interventions from January 2015 to December 2015, CONUT score $\geq 8$ was identified as an independent risk factor associated with NST intervention outcomes and the presence of PPN was secondly associated with NST intervention outcomes [18]. However, in this study, the presence of PPN was identified as an independent risk factor associated with NST intervention outcomes, and \%TEE $<66.0 \%$ and GNRI score $<70.0$ were secondly identified as risk factors. The results of this study appear to be more valid than the results of our previous study, because the number of participants in this study was two times that of our previous study and time concept was considered in this study.

PPN is primarily used when nutritional supply via oral or tube feeding is not sufficient, regardless of the intestine function. Therefore, the patients who received PPN at the time of NST enrollment had severe disease that prevented them from receiving adequate nutrients via oral or tube feeding. Moreover, the intensive and short-term nutritional care by the NST did not improve patients' nutritional status.

In some previous studies, which focused on \% TEE, nutritional management of patients was performed in the intensive care unit [19-21], although the background of the individuals was different from that of the individuals in this study, such as disease condition, vital signs, and methods for supplying nutrients. \%TEE or the energy intake was significantly associated with mortality or the length of hospital stay in previous studies. Consistent with this study, the previous study, which focused on the treatment of elderly undernourished patients in the general ward, showed that \%TEE was associated with mortality within 30 days [22]. In the nutritional management, it is essential to attempt to fill \%TEE. However, it is often difficult for elderly patients to increase their \%TEE, because elderly individuals have decreased dietary intake or lapse into undernutrition because of physical, mental, and social factors [2327]. The value of $\%$ TEE $66.0 \%$, validity of which is shown in this study, would be a potential reference index in nutritional management for elderly patients.

Based on the original method, patients with GNRI score $<82.0$ were considered to be extremely undernourished [17]. However, the cut-off value was 70.0, and validity was determined in this study. Therefore, elderly patients with GNRI score $<70.0$ were considered to be extremely undernourished at the time of NST enrollment. Thus, the nutritional status of patients who were admitted in an acute-care hospital, where the length of stay is limited, did not improve. In addition, while the CONUT and PNI scores were calculated based on laboratory data only, the formula for calculating the GNRI score includes body weight, which is obtained via a physical examination. Increasing a patient's body weight over a short period is more challenging than improving laboratory test results, including Alb levels. For example, the supplemental oral feeding of enteral nutrients significantly increased the body weight of patients with dementia [28]. However, the duration of the intervention was 12 weeks (medium-term treatments). Thus, when the effect of nutritional intervention over a short period was predicted using the GNRI score, the results showed that it was difficult for 
Table 4. Results of the Univariate and Multivariate Cox Proportional Hazard Analyses of the NST Intervention Outcomes

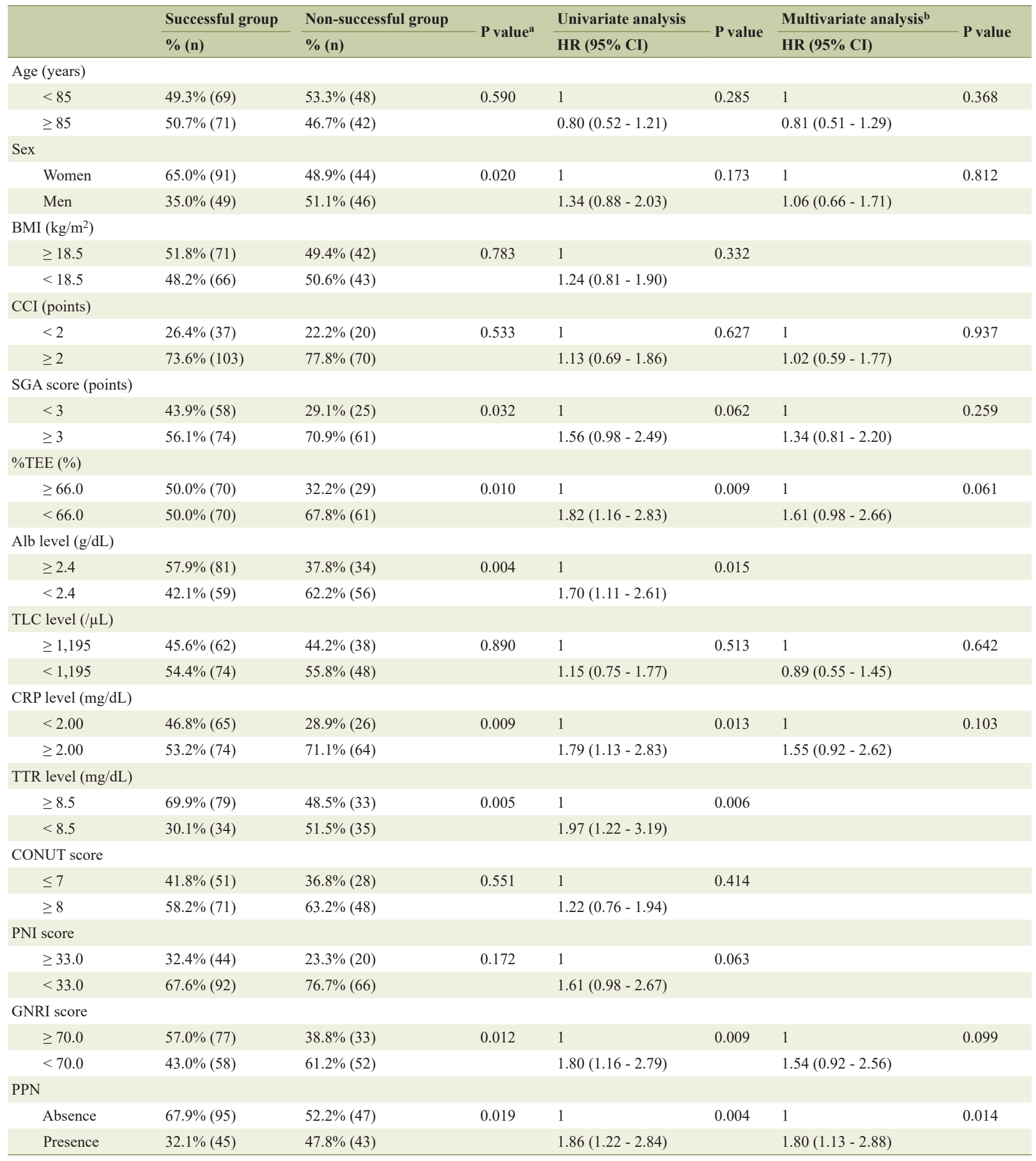

aFisher's exact test. 'bodel included age, sex, CCI score, SGA score, \%TEE, TLC, CRP, GNRI score, and PPN. HR: hazard ratio; Cl: confidence interval; BMI: body mass index; CCl: Charlson Comorbidity Index; SGA: subjective global assessment; \%TEE: the energy fill rate to total energy expenditure; Alb: albumin; TLC: total lymphocyte count; CRP: C-reactive protein; TTR: transthyretin; CONUT: controlling nutritional status; PNI: Onodera's prognostic nutritional index; GNRI: geriatric nutritional risk index; PPN: peripheral parental nutrition. 
patients with GNRI score $<70.0$, who were extremely undernourished, to recover from their nutritional condition.

In this study, at the time of NST enrollment, the presence of PPN, \% TEE $<66.0 \%$ and GNRI score $<70.0$ were considered independent risk factors associated with NST intervention outcomes in elderly undernourished individuals. The patients who exhibited these risk factors required some nutritional intervention. In almost all disease conditions, oral or tube feeding is highly prioritized compared to parenteral nutrition because it is widely accepted that "if the gut works, use it" [29]. The incidence of bacterial translocation or infectious complications caused by enteral nutrition is lower than that caused by parenteral nutrition $[30,31]$. However, even after considering the risk factors, patients must receive adequate nutrients via parenteral nutrition, thus enhancing the recovery of elderly patients with PPN, \% TEE $<66.0 \%$, or GNRI score $<70.0$ over a short period. In fact, the incidence of infection will not increase if adequate nutrients are provided via TPN [32]. Some nutrition care is also required for elderly individuals who are recuperating at their homes, nursing homes, or in long-term care facilities, so that they do not develop adverse conditions, which are the presence of PPN, \%TEE $<66.0 \%$, and GNRI score $<70.0$, when they undergo emergency hospitalization.

This was a single-center study, which is a limitation of our study. Future multicenter studies with a prospective cohort must be performed in higher level hospitals in urban areas to validate the effects of the risk factors identified in the present study.

\section{Conclusions}

Our results showed that the presence of PPN, \%TEE $<66.0 \%$, and GNRI score $<70.0$ are risk factors associated with NST intervention outcomes in elderly undernourished individuals. The patients who meet criteria, which are the presence of PPN, $\%$ TEE $<66.0 \%$, and GNRI score $<70.0$, will require new nutritional intervention, in addition to the nutrition therapy by a traditional NST. Elderly individuals should also receive some nutrition care because they have been recuperating at their home or in long-term care facilities, to avoid developing adverse conditions such as the presence of PPN, \% TEE $<66.0 \%$, and GNRI score $<70.0$.

\section{Acknowledgments}

The authors are grateful to the participants and the hospital members.

\section{Financial Disclosure}

No funding was received for this study.

\section{Conflict of Interest}

The authors declare no conflict of interest.

\section{Informed Consent}

Informed consent was obtained from each participant.

\section{Author Contributions}

$\mathrm{MH}, \mathrm{CM}, \mathrm{KS}, \mathrm{YY}$ and DH contributed to study conception and design; $\mathrm{MH}, \mathrm{YO}, \mathrm{CK}$ and TF contributed to data acquisition; $\mathrm{MH}, \mathrm{CM}, \mathrm{KS}$, and DH contributed to analysis and interpretation of the data and drafting and revision of the manuscript; All authors have approved the final version of the manuscript.

\section{References}

1. Persson MD, Brismar KE, Katzarski KS, Nordenstrom J, Cederholm TE. Nutritional status using mini nutritional assessment and subjective global assessment predict mortality in geriatric patients. J Am Geriatr Soc. 2002;50(12):1996-2002.

2. Odlund Olin A, Koochek A, Ljungqvist O, Cederholm T. Nutritional status, well-being and functional ability in frail elderly service flat residents. Eur J Clin Nutr. 2005;59(2):263-270.

3. Antonelli Incalzi R, Landi F, Cipriani L, Bruno E, Pagano F, Gemma A, Capparella O, et al. Nutritional assessment: a primary component of multidimensional geriatric assessment in the acute care setting. J Am Geriatr Soc. 1996;44(2):166-174.

4. Guigoz Y, Vellas B, Garry PJ. Assessing the nutritional status of the elderly: The Mini Nutritional Assessment as part of the geriatric evaluation. Nutr Rev. 1996;54(1 Pt 2):S59-65.

5. Dudrick SJ, Wilmore DW, Vars HM, Rhoads JE. Longterm total parenteral nutrition with growth, development, and positive nitrogen balance. Surgery. 1968;64(1):134142.

6. Traeger SM, Williams GB, Milliren G, Young DS, Fisher M, Haug MT, 3rd. Total parenteral nutrition by a nutrition support team: improved quality of care. JPEN J Parenter Enteral Nutr. 1986;10(4):408-412.

7. Fettes SB, Lough M. An audit of the provision of parenteral nutrition in two acute hospitals: team versus nonteam. Scott Med J. 2000;45(4):121-125.

8. Johansen N, Kondrup J, Plum LM, Bak L, Norregaard P, Bunch E, Baernthsen H, et al. Effect of nutritional support on clinical outcome in patients at nutritional risk. Clin Nutr. 2004;23(4):539-550.

9. Powers DA, Brown RO, Cowan GS, Jr., Luther RW, Sutherland DA, Drexler PG. Nutritional support team vs nonteam management of enteral nutritional support in a Veterans Administration Medical Center teaching hospital. JPEN J Parenter Enteral Nutr. 1986;10(6):635-638.

10. Faubion WC, Wesley JR, Khalidi N, Silva J. Total parenteral nutrition catheter sepsis: impact of the team approach. JPEN J Parenter Enteral Nutr. 1986;10(6):642645. 
11. Charlson ME, Pompei P, Ales KL, MacKenzie CR. A new method of classifying prognostic comorbidity in longitudinal studies: development and validation. J Chronic Dis. 1987;40(5):373-383.

12. Long CL, Schaffel N, Geiger JW, Schiller WR, Blakemore WS. Metabolic response to injury and illness: estimation of energy and protein needs from indirect calorimetry and nitrogen balance. JPEN J Parenter Enteral Nutr. 1979;3(6):452-456.

13. Japanese Society for parenteral and Enteral Nutrition. Practical guidelines for parenteral and enteral nutrition. Tokyo: Nankodo Co., Ltd., 2004. p.11. (In Japanease).

14. Ignacio de Ulibarri J, Gonzalez-Madrono A, de Villar NG, Gonzalez P, Gonzalez B, Mancha A, Rodriguez F, et al. CONUT: a tool for controlling nutritional status. First validation in a hospital population. Nutr Hosp. 2005;20(1):38-45.

15. Gonzalez-Madrono A, Mancha A, Rodriguez FJ, Culebras J, de Ulibarri JI. Confirming the validity of the CONUT system for early detection and monitoring of clinical undernutrition: comparison with two logistic regression models developed using SGA as the gold standard. Nutr Hosp. 2012;27(2):564-571.

16. Onodera T, Goseki N, Kosaki G. [Prognostic nutritional index in gastrointestinal surgery of malnourished cancer patients]. Nihon Geka Gakkai Zasshi. 1984;85(9):10011005.

17. Bouillanne O, Morineau G, Dupont C, Coulombel I, Vincent JP, Nicolis I, Benazeth S, et al. Geriatric Nutritional Risk Index: a new index for evaluating at-risk elderly medical patients. Am J Clin Nutr. 2005;82(4):777-783.

18. Hiramatsu M, Momoki C, Kato K, Yasui Y, Oide Y, Kaneishi C, Mori A, et al. Exploration of nutrition support team outcome predictor for elderly. J Nutri Med Diet Care. 2017;3(2):1-7.

19. Yun TJ, Jung YP, Jiyeon J, Myung JK, Seung HL and Jae GL. Association of inadequate caloric supplementation with 30-day mortality in critically III postoperative patients with high modified NUTRIC score. Nutrients. 2018;10(11):1589.

20. Higashibeppu N, Sanui M, Sobue K and Heyland DK. Association between greater energy intake and lower mortality in critically ill patients in the Japanese ICU. J
Jpn Soc Intensive Care Med. 2019;26:25-27. (In Japanease).

21. Heyland DK, Cahill N, Day AG. Optimal amount of calories for critically ill patients: depends on how you slice the cake! Crit Care Med. 2011;39(12):2619-2626.

22. Sanson G, Bertocchi L, Dal Bo E, Di Pasquale CL, Zanetti M. Identifying reliable predictors of protein-energy malnutrition in hospitalized frail older adults: A prospective longitudinal study. Int J Nurs Stud. 2018;82:40-48.

23. Clarkston WK, Pantano MM, Morley JE, Horowitz M, Littlefield JM, Burton FR. Evidence for the anorexia of aging: gastrointestinal transit and hunger in healthy elderly vs. young adults. Am J Physiol. 1997;272(1 Pt 2):R243-248.

24. Berthelemy P, Bouisson M, Vellas B, Moreau J, Nicole $\mathrm{V}$, Albarede JL, Ribet A. Postprandial cholecystokinin secretion in elderly with protein-energy undernutrition. J Am Geriatr Soc. 1992;40(4):365-369.

25. Boumendjel N, Herrmann F, Girod V, Sieber C, Rapin $\mathrm{CH}$. Refrigerator content and hospital admission in old people. Lancet. 2000;356(9229):563.

26. Wilson MM, Vaswani S, Liu D, Morley JE, Miller DK. Prevalence and causes of undernutrition in medical outpatients. Am J Med. 1998;104(1):56-63.

27. Vetta F, Ronzoni S, Taglieri G, Bollea MR. The impact of malnutrition on the quality of life in the elderly. Clin Nutr. 1999; 18(5):259-267.

28. Wouters-Wesseling W, Wouters AE, Kleijer CN, Bindels JG, de Groot CP, van Staveren WA. Study of the effect of a liquid nutrition supplement on the nutritional status of psycho-geriatric nursing home patients. Eur J Clin Nutr. 2002;56(3):245-251.

29. Livingston A, Seamons C, Dalton T. If the gut works use it. Nurs Manage. 2000;31(5):39-42.

30. Alexander JW. Nutrition and bacterial translocation. 1990;JPEN 14:170S-174S.

31. Moore FA, Feliciano DV, Andrassy RJ, McArdle AH, Booth FV, Morgenstein-Wagner TB, Kellum JM, Jr., et al. Early enteral feeding, compared with parenteral, reduces postoperative septic complications. The results of a metaanalysis. Ann Surg. 1992;216(2):172-183.

32. Jeejeebhoy KN. Total parenteral nutrition: potion or poison? Am J Clin Nutr. 2001;74(2):160-163. 